\title{
Green Radio Technique for Energy Efficient Wireless Networks with Hybrid Solar Wind System
}

\author{
T.Sridevi" ${ }^{\# 1}$ \\ Assistant professor, Sri Sairam institute of technology, Chennai \\ Corresponding Author: T.Sridevi
}

\begin{abstract}
Nowadays the number of users and the use of telecommunication systems are increasing rapidly and this result in greater demands on energy usage. Based on the extensive Life-cycle assessment (LCA) conducted by various network operators, it is learned that energy consumption in the usage phase of its radio access networks is the most imminent factor relating to impact on the environment. The amount of carbon emission is increasing in communication system, in parallel with increase in mobile consumers due to the increased use of DG. And it is also observed that current wireless networks are not energy-efficient, mainly the base stations (BS). This alarming growth in mobile users and increase in carbon emission forces us to use higher data rate mobile broadband. There is a need for restructuring of existing network architecture. We need to keep controlling system in every base station for switching purpose. Our project restructures the existing mobile network by controlling the ON and OFF state of the tower with respect to the number of users at demand and generation of green energy from a hybrid solar-wind system is done to supply the mobile network. By implementing our project, we can achieve an energy efficient mobile network by reducing energy consumed by tower and by generating green energy leading to reduction in carbon emission.

Keywords: carbon Emission, Wireless Mobile Communication, Green radio technique, PIC microcontroller, reactive power.
\end{abstract}

\section{INTRODUCTION}

In our country we have a huge demand for power. Energy saving is the most important factor. In recent years, there is a rapid growth in the number of mobile users and hence we use higher-data-rate mobile broadband. Electricity consumption attributed to information and communication technology is also increasing rapidly. The source for towers is based on the power requirement. A critical mobile network consumes 40-50MW approximately.In developing countries direct electricity connections are not readily available, so an operator must go for generator as the source of power. For continuous and instantaneous operation, conventional types of energy resources are preferred. Diesel generator is preferred over other types of generators. A typical diesel generator, used for supplying power for communication purpose, consumes one million gallon (1MG) of diesel per day. This results in emission of huge amount of carbon-dioxide (CO2). The impact due to several million towers installed in a country is huge. Thus, there is a need on environmental grounds to reduce the energy requirements of radio access networks. The architectural operation of present scenario is not energy-efficient. It does not account for user frequency (i.e. number of mobile users) in an area. All the towers in that area is turned ON irrespective of the user frequency. This leads to enormous amount of fuel wastage. Now considering one single tower, power is not only used for the signal transmission process (which consumes major portion of power), but also for running the cooling unit of generator and light indicator present along with lightning arrestor. All these instruments consume more power than that is actually required. From an operator's perspective, reducing energy consumption will also translate to lower Operating Expenditure (OPEX) costs. Reducing carbon emissions and OPEX for wireless cellular networks are two key reasons behind the development of the Mobile Green Radio program. The Green Radio project is pursuing energy efficient approach from two different perspectives. The first is to examine alternatives to the existing cellular network structures to reduce energy consumption. The second approach is to use a hybrid of solar-wind system for generating energy for supplying the wireless network as additional to the diesel generators used. In this paper novel method has been established for Energy-Efficient wireless network and to generate green energy to support the DG thereby achieving EcoBalance in nature by reducing carbon emission. 


\section{WIRELESS MOBILE COMMUNICATION NETWORKS} 2.1 PRESENT SCENARIO

Since 2006, data traffic on wireless networks has grown by approximately $400 \%$ and is expected to continue to increase rapidly in the coming years. The widespread use of complex, spectrum efficient techniques to support such high data volumes, the demand for higher data rates and the ever-increasing number of wireless users translate to rapidly rising power consumption. Currently consuming $3 \%$ of the energy and causing $2 \%$ of the $\mathrm{CO} 2$ emissions globally, the Information \& Communication Technology (ICT) industries are facing an increase in associated energy consumption of $16-20 \%$ per year. Tons and tons of litres of diesel are consumed by mobile towers. India has around 3.1 lakh towers, of which 70 percent are located in rural areas where grid connected electricity is not available. As a result, 60 percent of the towers are powered by diesel generators which produce a total of 5.3 million litres of carbon dioxide every year[3]. Many so-called "standby" generator sets installed at urban cell towers are in fact running for several hours every day. With the sustained rise of global energy prices, the fuel costs of running these diesel, natural gas or propane generators are a major piece of the Total Cost of Ownership (TCO) for these cell towers.

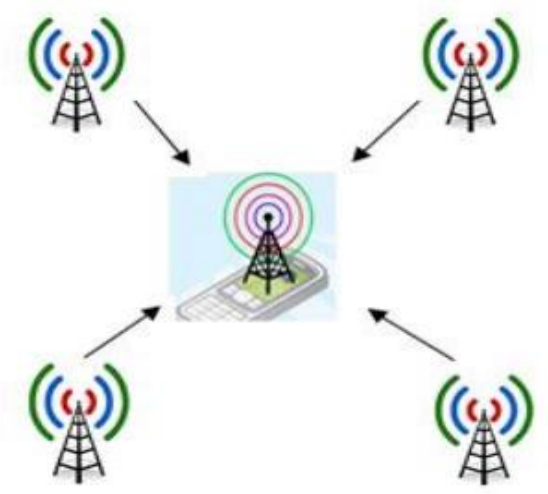

Fig 2.1 Current scenario of communication network

\subsection{IMPACT AND CONSEQUENCES}

Because of the continuous operation, more fuel is consumed. This results in high power consumption rate, high $\mathrm{CO} 2$ emission rate, depletion of fossil fuels, higher operating expenditure (OPEX) costs, many other ecological imbalances ( like acid rain, green house effect, ozone layer depletion etc..). In order to aid the required power consumption a hybrid solar wind system is used in addition to power consumption reduction in the existing system by a control mechanism in our proposed system.

\section{PROPOSED SYSTEM CONFIGURATION}

\subsection{Green radio technique}

The proposed system consists of a PIC (Peripheral Integral Controller) to monitor and control various parameters like voltage, current, responder frequency, light intensity, temperature, humidity and diesel generator are sensed and given to the eight A/D pins of PIC. The switching of these parameters, when it exceeds the limit, is taken care of by relays.

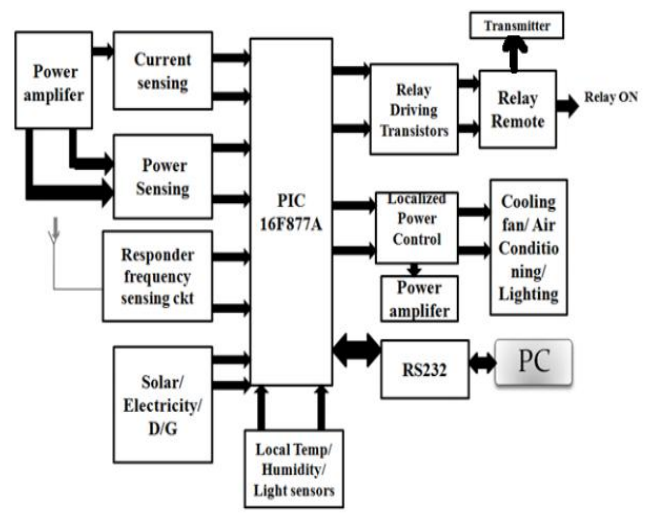

Fig 3.1 Block Diagram of Green Radio Technique

In this technique, the mobile-communication tower in an area is turned ON, based on the frequency of users present in that area. In general, within a predefined control area, there will be multiple towers operating (of same network type). And each tower has pre-defined user-strength capacity, up to which it can operate. Thus, based on the user strength in a region, the number of towers in that particular region is turned ON and remaining towers are kept in IDLE STATE.

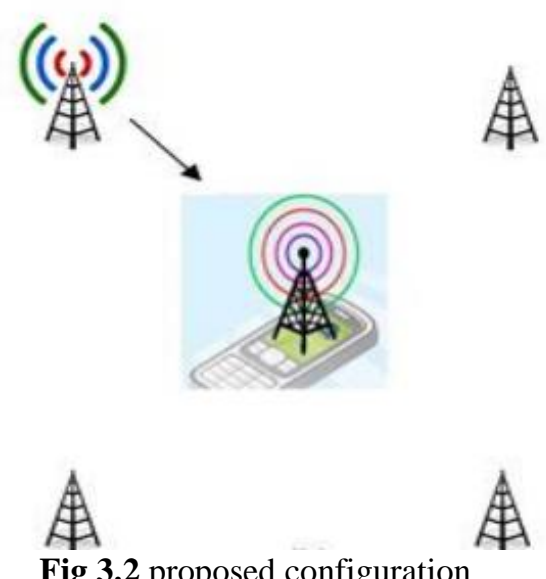

Apart from implementing this configuration, we monitor certain parameters, which can help in reducing energy consumption, they are Responder Frequency, Cooling-Fan, Air Conditioner, and Light 
Indicator. Every tower has a lightning conductor, along with Light Indicator. This light glows throughout the day, which is not necessary. Similarly, we have Cooling-Fan and Air Conditioner running round the clock. Thus a tower consumes power to run all these accessories, apart from power used for transmitting signal. So, this technology proposes a novel method to implement a corrective measure, which will ensure optimum utilization of energy resources. In order to control these parameters automatically, we use microcontroller based embedded system. The PIC micro-controller is used to do the control job and send the signal to a computer. Here, PIC micro-controller is interfaced with Integrated chips (ICs). Each of those ICs perform different task whose output is given to PC through PIC micro controller. We use LDR to monitor the light intensity around the tower to determine day-break and night. We use two thermistors to determine temperature and moisture content. There is 9 standard value fixed for all these parameters. When it deviates from that switching action takes place through relay circuits. In our model, we use four relays to monitor each of temperature, humidity, light intensity and frequency. For example light intensity above 1000 lumens is considered to be day-break, if not, its night. Hence light indicator will be turned $\mathrm{ON}$ automatically during night time. Similarly, based on Temperature and Humidity of the generator room, Air-Conditioner and Cooling-Fan will operate respectively. This is the basic operation of our model.

\section{HYBRID SOLAR WIND SYSTEM}

Both wind power and solar power will be acquired simultaneously and the merged together using synchronization circuits and one common power will be send out for charging the batteries . To collect wind energy, we are using wind turbines made up of D.C generators with mechanical model. The wind turbine is capable of rotating for small amount of wind change on the ambient. The maximum output of the turbine will be $24 \mathrm{~W}$ and can work continuously, this can further be enhanced to larger value for real time implementation. To acquire solar energy, we use photo-voltaic cell made up of amorphous silicon module, very rigid, high thermal stability and non-fadable device will be utilized for this application. The energy received in terms of power will be $12 \mathrm{~W}$.

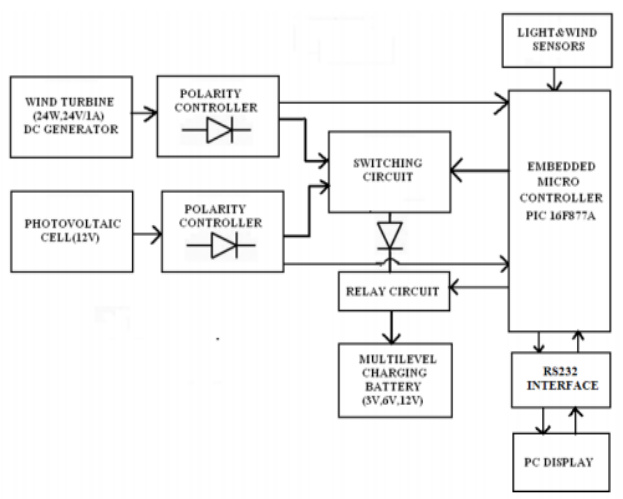

Fig 4.1 Block diagram of Hybrid Solar Wind system.

The photo-voltaic cell will be installed down to the wind turbine and works even with maximum light. All these data's will be presented on the PC screen for further research as database. Acquired voltage will be displayed on the PC screen as numerical value.

\section{PIC (PERIPHERAL INTEGRAL CONTROLLER)}

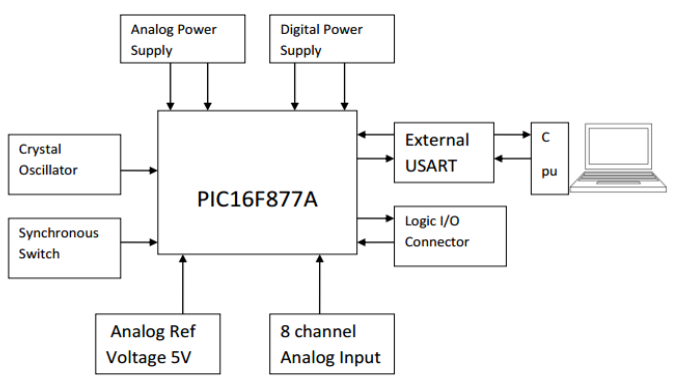

Fig 5.1 block diagram of peripheral integral controller

Peipheral integral controllers (PICs) are popular due to their low cost, availability, large user base, extensive collection of application notes, availability of low cost or free development tools, and serial programming (and reprogramming with flash memory) capability. The 40 pin IC has five I/O ports, fifteen interrupts and eight $\mathrm{A} / \mathrm{D}$ input channels. It is used to control and monitor different parameters like Temperature, Humidity, Light Intensity, Responder Frequency, UPS, Diesel Generator, Voltage and Current. All these are given to the 8 analog input channels and corresponding control action is taken if there is any violation of pre-defined constraint.

\section{RS-232 INTERFACE}

\subsection{RS-232 CONVERTER}

RS - 232 Converter is used for PC application. The serial port converts the output of PC, 
which is in parallel form into serial data for transmission. The external converter Quadruples the incoming 5V (from PIC) and gives $20 \mathrm{~V}$ output. That is $-10 \mathrm{~V}$ to $+10 \mathrm{~V}$. Similarly the incoming $20 \mathrm{~V}$ from (from PC) is reduced as $5 \mathrm{~V}$ by the converter.

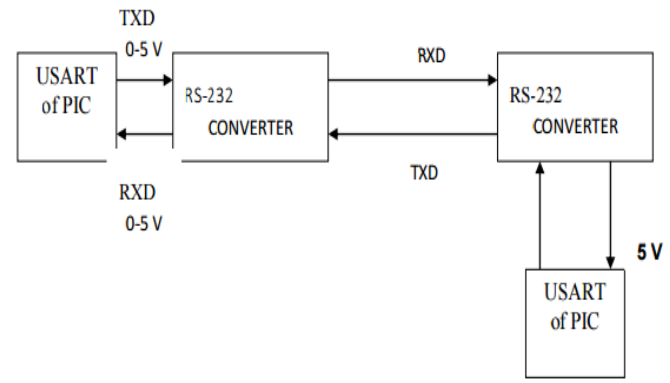

Figure 6.1 RS - 232 Converter operations 6.2 MAX 232

The Max 232 is a dual RS-232 receiver / transmitter that meet all EIA RS232C specifications while using only a $\pm 5 \mathrm{~V}$ power supply. It has 2 onboard charge pump voltage converters which generate $+10 \mathrm{~V}$ and $-10 \mathrm{~V}$ power supplies from a single $5 \mathrm{~V}$ power supply. It has four level translators, two of which are RS232 transmitters that convert TTL $\backslash$ CMOS input levels into + 9V RS232 outputs. The other two level translators are RS232 receivers that convert RS232 inputs to 5V TTLICMOS output level. These receivers have a nominal threshold of $1.3 \mathrm{~V}$, a typical hysteresis of $0.5 \mathrm{~V}$ and can operate upto $+30 \mathrm{~V}$ input.

1. Suitable for all RS232 communications and $+12 \mathrm{~V}$ power supplies required. TXD 0-5 V RXD RS-232 CONVERTER RS-232 CONVERTER USART of PIC USART of PIC RXD 0-5 V TXD 5 V

2. Voltage quadrapular for input voltage upto5.5V (used in power supply Section of computers, peripherals, and modems).

Three main sections of MAX232 are

1. A dual transmitter

2. A dual receiver

$3.5 \mathrm{~V}$ to $\pm 10 \mathrm{~V}$ dual charge pump voltage converter

\section{SOLAR PANEL}

Solar panel refers to a panel designed to absorb the sun's rays as a source of energy for generating electricity or heating. A photovoltaic (PV) module is a packaged, connect assembly of typically $6 \times 10$ photovoltaic solar cells. Photovoltaic modules constitute the photovoltaic array of a photovoltaic system that generates and supplies solar electricity in commercial and residential applications. Each module is rated by its DC output power under standard test conditions (STC), and typically ranges from 100 to 365 watts.

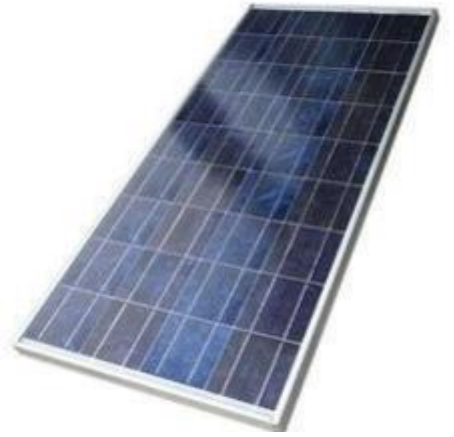

Fig 7.1 solar panel

The efficiency of a module determines the area of a module given the same rated output - an $8 \%$ efficient 230 watt module will have twice the area of a $16 \%$ efficient 230 watt module. There are a few commercially available solar modules that exceed $22 \%$ efficiency and reportedly also exceeding $24 \%$. A single solar module can produce only a limited amount of power; most installations contain multiple modules. A photovoltaic system typically includes an array of photovoltaic modules, an inverter, a battery pack for storage, interconnection wiring, and optionally a solar tracking mechanism.

\section{WIND TURBINE}

A wind turbine is a device that converts the wind's kinetic energy into electrical power. Wind turbines are manufactured in a wide range of vertical and horizontal axis types. The smallest turbines are used for applications such as battery charging for auxiliary power for boats or caravans or to power traffic warning signs. Slightly larger turbines can be used for making contributions to a domestic power supply while selling unused power back to the utility supplier via the electrical grid. Arrays of large turbines, known as wind farms, are becoming an increasingly important source of intermittent renewable energy and are used by many countries as part of a strategy to reduce their reliance on fossil fuels.

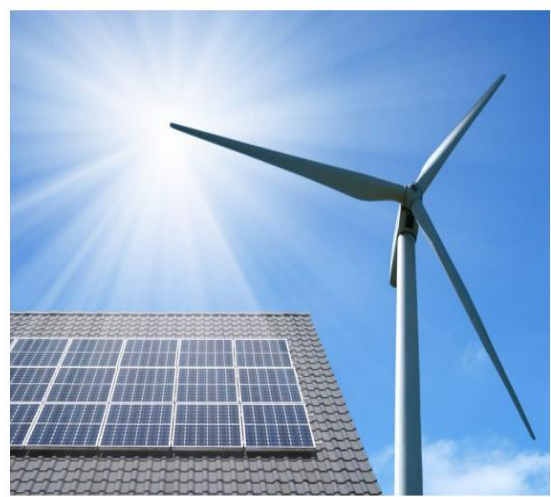

Fig 8.1 wind turbine with solar panel 


\section{SIMULATION RESULTS}

The simulation for the project is done using Visual Basic and the PCB is designed using OrCAD software.

\subsection{ABOUT VISUAL BASIC}

Visual Basic is a third-generation eventdriven programming language and integrated development environment (IDE) from Microsoft for its Component Object Model (COM) programming model first released in 1991 and declared legacy during 2008. Microsoft intended Visual Basic to be relatively easy to learn and use. Visual Basic was derived from BASIC, a user-friendly programming language designed for beginners, and it enables the rapid application development (RAD) of graphical user interface (GUI) applications, access to databases using Data Access Objects, Remote Data Objects, or ActiveX Data Objects, and creation of ActiveX controls and objects. Programs written in Visual Basic can also use the Windows API, which requires external function declarations. The final release was version 6 in 1998 (now known simply as Visual Basic). On April 8, 2008 Microsoft stopped supporting Visual Basic 6.0 IDE. The Microsoft Visual Basic team still maintains compatibility for Visual Basic 6.0 applications on Windows Vista,

\section{CONCLUSION}

This paper proposes new advanced usage of FACTS devices such as UPFC which can do both shunt and series compensation. Also it differentiates the usage of FACTS devices UPFC and STATCOM. The proposed system using UPFC is discussed has the following advantages, can compensate both voltage and current simultaneously independently, this also reduces the line losses in a power system. This method also enhances the load voltage profile which protects the load from various damages. Due to improved techniques the harmonic distortions is minimized. The usage of UPFC in a power system improves the power factor in the system.

\section{REFERENCES}

[1] Z. Shu, X. He, Z. Wang, D. Qiu, and Y. Jing, "Voltage balancing approaches for diodeclamped multilevel converters using auxiliary capacitor-based circuits," IEEE Trans. Power Electronics, vol. 28, no. 5, pp. 2111-2124, 2013.
Windows Server 2008 including R2, Windows 7, Windows 8, Windows 8.1, Windows Server 2012 and Windows 10 through its "It Just Works" program. In 2014, some software developers still preferred Visual Basic 6.0 over its successor, Visual Basic .NET. In 2014 some developers lobbied for a new version of Visual Basic 6.0. In 2016, Visual Basic 6.0 won the technical impact award at The 19th Annual D.I.C.E. Awards. A dialect of Visual Basic, Visual Basic for Applications (VBA), is used as a macro or scripting language within several Microsoft applications, including Microsoft Office.

\subsection{ORCAD PCB DESIGNER}

OrCAD PCB Designer is a printed circuit board designer application, and part of the OrCAD circuit design suite. PCB Designer includes various automation features for PCB design, board-level analysis and design rule checks (DRC). 36 he PCB design may be accomplished by manually tracing PCB tracks, or using the Auto-Router provided. Such designs may include curved PCB tracks, geometric shapes, and ground planes. PCB Designer integrates with OrCAD Capture, using the component information system (CIS) to store information about a certain circuit symbol and its matching PCB footprint.

[2] J. Verveckken, F. Silva, D. Barros, and J. Driesen, "Direct power control of series converter of unified power-flow controller with three-level neutral point clamped converter," IEEE Trans. Power Delivery, vol. 27, no. 4, pp. 1772-1782, 2012.

[3] J.-F. Moon and J.-S. Kim, "Voltage sag analysis in loop power distribution system with sfcl," IEEE Trans. Applied Superconductivity, vol. 23, no. 3, pp. 5601504-5601504, 2013.

[4] C.-J. Chou and C.-W. Liu, "Assessment of risks from ground fault transfer on closedloop hv underground distribution systems with cables running in a common route," IEEE Trans. Power Delivery, vol. 28, no. 2, pp. 1015-1023, 2013

[5] M. Rezaei and A. Huang, "Ultra fast protection of radial and looped electric power grid using a novel solid-state protection device," in IEEE Energy Conversion Congress and Exposition (ECCE), 2012, pp. 610-614.

T.Sridevi. "Green Radio Technique for Energy Efficient Wireless Networks with Hybrid Solar Wind System ." International Journal of Engineering Research and Applications (IJERA) 7.7 (2017): 67-71. 